Discrete Comput Geom 38:177-187 (2007)

DOI: $10.1007 / \mathrm{s} 00454-007-1332-9$

\title{
Packing Cones and Their Negatives in Space*
}

\author{
Imre Bárány ${ }^{1}$ and Jiří Matoušek ${ }^{2}$ \\ ${ }^{1}$ Rényi Institute of Mathematics, Hungarian Academy of Sciences, \\ PO Box 127, 1364 Budapest, Hungary \\ barany@math-inst.hu \\ and \\ Department of Mathematics, University College London, \\ Gower Street, London WC1E 6BT, England \\ ${ }^{2}$ Department of Applied Mathematics and \\ Institute of Theoretical Computer Science (ITI), Charles University, \\ Malostranské nám. 25, 11800 Praha 1, Czech Republic \\ matousek@kam.mff.cuni.cz
}

\begin{abstract}
Let $C$ be a cone in $\mathbf{R}^{3}$ whose base $B$ is a planar convex body in a horizontal plane $\pi$ and whose tip is a point $v \notin \pi$. Let $\mathcal{C}$ be a packing formed by translates of $C$ and $-C$ in $\mathbf{R}^{3}$. We exhibit an explicit constant $c>0$ such that the density of any such $\mathcal{C}$ is smaller than $1-c$, answering a question of Wlodek Kuperberg.
\end{abstract}

\section{Introduction and Main Result}

Let $C$ be a cone, over a planar convex set $B$, in $\mathbf{R}^{3}$ and let $\mathcal{C}$ be a packing consisting of translates of $C$ and $-C$ (no rotations allowed). Kuperberg [5] proved several years ago that the density $\delta(\mathcal{C})$ of $\mathcal{C}$ is less than 1 (for the reader's convenience, we outline a short proof at the end of this section). This immediately implies the existence of a constant $c>0$ such that $\delta(\mathcal{C}) \leq 1-c$ for every $C$ and every packing $\mathcal{C}$ of translates of $C$ and $-C$. Indeed, if $\sup _{C, \mathcal{C}} \delta(\mathcal{C})=1$, then one can choose a convergent subsequence of the cones such that the limiting cone tiles the space. However, then the density of the corresponding packing is 1 .

This argument cannot give any explicit value for $c$. That is why Kuperberg [5] raised the following problem: Find an explicit constant $c>0$ such that for every cone $C$, every packing by translates of $C$ and $-C$ has density less than $1-c$. The aim of this paper is to give such an explicit constant.

\footnotetext{
* Imre Bárány was supported by Hungarian National Foundation Grants T 046246 and T 037846.
} 
Here a cone $C$ is simply the convex hull of the base $B$ and the tip $v$, where $B$ is a convex compact set of nonzero area lying in a two-dimensional plane $\pi$, and $v \notin \pi$ is a point in $\mathbf{R}^{3}$.

Theorem 1.1. There is an explicit constant $c>0$ such that for every cone $C \subset \mathbf{R}^{3}$, every packing by translates of $C$ and $-C$ has density smaller than $1-c$.

Remarks. Our proof actually works for larger class of packings, with the same constant $c$. Namely, let $\mathcal{F}$ be the family of all cones in $\mathbf{R}^{3}$ with tips at $(0,0,1)$ or at $(0,0,-1)$ and with bases $B$ in the plane $z=0$ such that $B$ contains the horizontal unit disk centered at the origin and is contained in the concentric disk of radius 2. Let $\mathcal{C}$ be a packing of translates of cones in $\mathcal{F}$. Then the density of $\mathcal{C}$ is at most $1-c$. For this remark we are indebted to Wlodek Kuperberg.

Our method gives an extremely small value for $c$. (We have not tried to optimize the constants in the proofs.) It is very easy to see that if the base $B$ tiles the plane, then there exists a packing $\mathcal{C}$ by translates of $C$ and $-C$ whose density is $\frac{2}{3}$. The best construction we know of is more than 100 years old and is due to Minkowski [6]. It is a lattice packing by translates of an octahedron with density $\frac{18}{19}$, showing that the constant in Theorem 1.1 satisfies $c \leq \frac{1}{19}$. Betke and Henk [1] proved that no lattice packing of octahedra can have a larger density.

Sketch of a Proof of $\delta(\mathcal{C})<1$. We assume that $\mathcal{C}$ is a packing of translates of $C$ and $-C$, and we show that $\delta(\mathcal{C})<1$, the result of Kuperberg. This, of course, is weaker than Theorem 1.1, but the proof is simple.

For contradiction we assume $\delta(\mathcal{C})=1$ and let $\mathcal{C}_{n}$ be a packing by translates of $C$ and $-C$ such that $\delta\left(\mathcal{C}_{n}\right)$ tends to 1 . Let $Q$ be a large cube. Then there are translated copies $Q_{n}$ of $Q$ such that, as $n$ goes to infinity,

$$
\sum_{C^{*} \in \mathcal{C}_{n}} \operatorname{Vol}\left(C^{*} \cap Q_{n}\right) \rightarrow \operatorname{Vol} Q .
$$

Translate $Q_{n}$ to $Q$ together with the $C^{*} \in \mathcal{C}_{n}$ that intersect $Q_{n}$. We get finite packings by translates of $C$ and $-C$ that cover $Q$ almost perfectly. One can choose a convergent subsequence of these packings, and the limiting packing $\mathcal{C}^{*}$ tiles $Q$. Then $C$ is a polytope. Let $C^{*}$ be a cone in $\mathcal{C}^{*}$ which is close to the center of $Q$, and let $T$ be a triangular facet of $C^{*}$ adjacent to the tip. Every point $p$ in the relative interior of $T$ is covered (besides $C^{*}$ ) by another cone $C(p) \in \mathcal{C}^{*}$. Further, $C^{*}$ and $C(p)$ are separated by the plane $\operatorname{aff}(T)$. Now if $C(p)$ is a translate of $C$, then $C(p) \cap \operatorname{aff}(T)$ is a vertex or an edge of $C(p)$. This implies that the translates of $C$ in $\mathcal{C}^{*}$ can only cover a small portion (of measure zero) of $T$. For the rest of the points $p \in T, C(p)$ is a translate of $-C$. Consequently, $p$ is covered by $-T$. However, that is impossible: a triangle $T$ cannot be covered by internally disjoint translates of $-T$.

Remark. There are several beautiful open questions about the density of packings of cones in $\mathbf{R}^{d}, d \geq 3$, some of them are very natural and look hard. We refer the interested reader to the forthcoming paper by Bezdek and Kuperberg [3] with the hope that it 
will be written up and published soon. Some information on these problems can also be found in [2].

\section{Preparations}

In this section we introduce notation and terminology, and state auxiliary lemmas needed in the proof.

We assume that the base $B$ of the cone $C$ lies in the horizontal plane $\pi$ containing the origin 0 . For a real number $x$ we let $\pi(x)$ be the plane parallel to $\pi$ at distance $|x|$ from $\pi=\pi(0)$, where $\pi(x)$ lies below $\pi$ for $x>0$ and above $\pi$ for $x<0$. This is opposite(!) to the usual convention for the position of the coordinate system, but we find our "reverse" convention more convenient in this paper.

Let $D \subset \pi$ be the unit disk centered at the origin. Since our problem is invariant under nondegenerate linear transformations, we can assume that $B$ is sandwiched between $\frac{1}{2} D$ and $D$, that is, $\frac{1}{2} D \subset B \subset D$ (by Löwner's theorem [4]). Similarly, we may assume that the tip $v$ of $C$ is above the origin and at distance 1 from it (so it lies in $\pi(-1)$ ). The sandwiching easily implies the following two facts, whose elementary proofs are omitted.

Fact 2.1. For every point $p$ on the boundary of $B \subset \pi$, the angle between $\pi$ and the line connecting $p$ and $v$ is between $45^{\circ}$ and $60^{\circ}$.

Fact 2.2. For every $p$ on the boundary of $B \subset \pi$, there is a wedge $K$ in the plane $\pi$ with apex at $p$ and of angle $60^{\circ}$ such that $K \cap\left(p+\frac{1}{2} D\right)$ is contained in $B$; see Fig. 1.

Let $C^{*}$ be a translated copy of $C$. We write $v\left(C^{*}\right)$ for its tip, $B\left(C^{*}\right)$ for its base, and we let $a\left(C^{*}\right)$ be the vertical coordinate of the base; that is, $B\left(C^{*}\right)$ lies in the plane $\pi\left(a\left(C^{*}\right)\right)$. So $a\left(C^{*}\right) \in[0,1]$ if and only if $C^{*}$ intersects $\pi$. For a translate $C_{i}$ of $C$ we simply write $a_{i}, B_{i}, v_{i}$ instead of $a\left(C_{i}\right), B\left(C_{i}\right), v\left(C_{i}\right)$.

We write $\operatorname{dist}\left(S_{1}, S_{2}\right)$ for the Euclidean distance between sets $S_{1}, S_{2} \subset \mathbf{R}^{3}$. Of course, the distance between $S_{1}$ and $S_{2}$ is the infimum of $\operatorname{dist}(x, y)$ with the infimum taken over all $x \in S_{1}$ and $y \in S_{2}$.

We need three simple lemmas for the proof of the main theorem.

Lemma 2.3 (Avoidance Lemma). Let $C_{1}$ and $C_{2}$ be disjoint translates of $C$, both intersecting $\pi$, and let $0 \leq a_{2} \leq a_{1} \leq 1$; see Fig. 2. Then

$$
\operatorname{dist}\left(\pi \cap C_{1}, \pi \cap C_{2}\right) \geq a_{2} \text {. }
$$

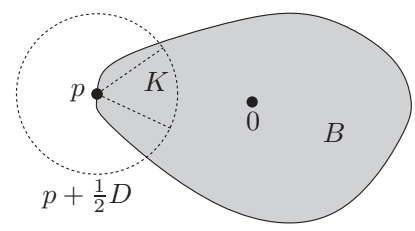

Fig. 1. Illustration to Fact 2.2. 


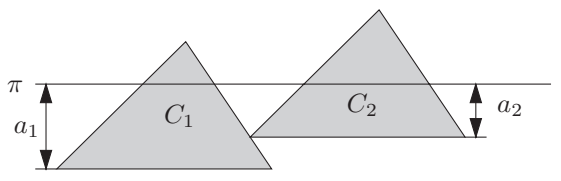

Fig. 2. The avoidance lemma.

For the next lemma and for the rest of the paper we set $r_{0}=\frac{1}{12}$.

Lemma 2.4 (Local Boundedness Lemma). Let $r D$ be the disk in $\pi$ of radius $r$ centered at 0 , where $r \in\left(0, r_{0}\right]$, and let $\mathcal{C}^{+}$be a packing of translates of $C$ (no-C allowed here). Then $r D$ intersects at most one cone from $\mathcal{C}^{+}$with $a\left(C^{*}\right) \geq 2 r$ and at most six cones with $a\left(C^{*}\right)<2 r$.

For $0 \leq h_{2}<h_{1}$ we let $\operatorname{Cyl}\left(r, h_{1}, h_{2}\right)$ denote the vertical cylinder of radius $r$ with axis passing through 0 and bounded from above by the plane $\pi\left(h_{2}\right)$ and from below by $\pi\left(h_{1}\right)$. Let $C_{0}$ denote the translate of $C$ whose tip is at the origin.

Lemma 2.5 (Special Cylinder Lemma). Let $\alpha$ be a sufficiently small positive real number, and let $\beta \in(0, \alpha)$. For every $R \in\left(0, r_{0}\right]$, and for every packing $\mathcal{C}^{+}$of translates of $C$ with $C_{0} \in \mathcal{C}^{+}$there is an $r$ with

$$
\left(\frac{2 \beta}{2+\alpha}\right)^{6} R \leq r \leq R
$$

such that $C_{0}$ is the only cone of $\mathcal{C}^{+}$intersecting the interior of $\mathrm{Cyl}(r, \alpha r, \beta r)$; see Fig. 3.

We use these lemmas in the proof of the main theorem. Their proofs are given in Section 5.

\section{One More Lemma and Proof of the Main Theorem}

We assume that $r \in\left(0, r_{0}\right], \alpha>0$, and $\beta \in(0, \alpha / 2]$ have been fixed. Let $Z$ be the cylinder $\operatorname{Cyl}(r, \alpha r, \beta r)$ and let $T$ be its axis, that is, the segment of the vertical line through 0 between the planes $\pi(\alpha r)$ and $\pi(\beta r)$. We also set $\gamma=\alpha-\beta$ and $\eta=\alpha^{2}$.

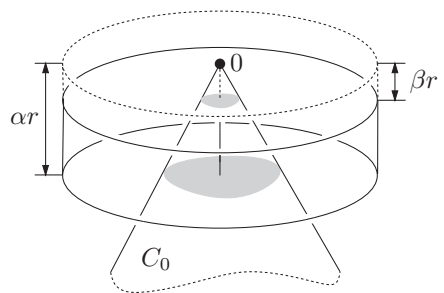

Fig. 3. Illustration to the special cylinder lemma. 
Lemma 3.1 (Main Lemma). Let $\mathcal{C}^{+}$be a packing of translates of $C$ in which each element is disjoint from $T$. Then

$$
\operatorname{Vol}\left(Z \backslash \bigcup \mathcal{C}^{+}\right) \geq \eta \operatorname{Vol} Z
$$

The proof is given in the next section. We are actually going to use the lemma for the translates of $-C$ in the given packing of $C$ and $-C$.

Proof of Theorem 1.1. We specify the parameters now, but we work with their numerical values only later. So let $\alpha=1 /(384 \pi)$, and $\beta=\gamma=\alpha / 2$. We choose $R=r_{0}=\frac{1}{12}$.

Lemma 2.5 applies to every positive cone $C_{i} \in \mathcal{C}$ and to the packing $\mathcal{C}^{+}$consisting of the positive cones in $\mathcal{C}$. This gives, for every $C_{i}$, a cylinder $Z_{i}=\operatorname{Cyl}\left(r_{i}, \alpha r_{i}, \beta r_{i}\right)+v_{i}$ that is intersected only by $C_{i}$ and possibly by some translates of $-C$ in $\mathcal{C}$, but by no cone in $\mathcal{C}^{+} \backslash\left\{C_{i}\right\}$.

We also note that all $r_{i}$ are larger than the fixed positive number

$$
\left(\frac{2 \beta}{2+\alpha}\right)^{6} R=\left(\frac{\alpha}{2+\alpha}\right)^{6} R
$$

and so

$$
\operatorname{Vol} Z_{i}=\pi \gamma r_{i}{ }^{3} \geq \pi \gamma\left(\frac{\alpha}{2+\alpha}\right)^{18} R^{3}=: c_{0} .
$$

The negative cones in $\mathcal{C}$ are disjoint from the axis of $Z_{i}$ because this axis is contained in $C_{i}$. Then the main lemma obviously can be applied to $Z_{i}$ and to the packing $\mathcal{C}^{-}$formed by the negative cones in $\mathcal{C}$. So the negative cones in $\mathcal{C}$ occupy at most a $1-\eta$ fraction of $Z_{i}$. The only positive cone intersecting $Z_{i}$ is $C_{i}$, and $\operatorname{Vol}\left(Z_{i} \cap C_{i}\right) \leq\left(\alpha r_{i}\right)^{3} \pi / 3$. Thus $\mathcal{C}$ altogether misses at least

$$
\eta \operatorname{Vol} Z_{i}-\frac{\pi}{3}\left(\alpha r_{i}\right)^{3}=\left(\eta-\frac{\alpha^{3}}{3 \gamma}\right) \operatorname{Vol} Z_{i}=\left(\eta-\frac{2 \alpha^{2}}{3}\right) \operatorname{Vol} Z_{i}=\frac{\eta}{3} \operatorname{Vol} Z_{i}
$$

of the volume of $Z_{i}$, since we have chosen $\eta=\alpha^{2}$.

Using the avoidance lemma (Lemma 2.3) it is easy to check that the cylinders $Z_{i}$ are disjoint. Consequently, for each positive cone $C_{i} \in \mathcal{C}^{+}$, an $\eta / 3$ fraction of the volume of the cylinder $Z_{i}$ is left uncovered by $\mathcal{C}$.

The same applies to the negative cones in $\mathcal{C}$ as well. Now when computing the density of $\mathcal{C}$, we consider a large cube $Q$ containing $n$ cones from the packing, with at least half of them positive, say. If $n \mathrm{Vol} C<\frac{1}{2} \mathrm{Vol} Q$, then the density in $Q$ is small, smaller than $\frac{2}{3}$ for Vol $Q$ sufficiently large, since the cones from $\mathcal{C}$ that intersect $Q$ but are not contained in $Q$ can cover only a small portion of $Q$. So we now suppose that $n \operatorname{Vol} C \geq \frac{1}{2} \operatorname{Vol} Q$. Then in the cylinders $Z_{i}$ corresponding to the positive cones from $\mathcal{C}$ contained in $Q$, a volume of at least

$$
\frac{n}{2} \frac{\eta}{3} c_{0}
$$


is uncovered by $\mathcal{C}$, while the volume of $Q$ is at most $2 n \operatorname{Vol} C \leq(2 \pi / 3) n$. This implies that $\mathcal{C}$ leaves an $\varepsilon$ fraction of $Q$ uncovered, where

$$
\varepsilon=\frac{\eta c_{0}}{4 \pi}=\frac{1}{8} \frac{\alpha^{21} R^{3}}{(2+2 \alpha)^{18}} \approx 5.327 \cdot 10^{-75} .
$$

Remark. By fine-tuning the parameters in this argument and in the proof of the main lemma it is possible to get $\varepsilon \approx 10^{-42}$. This is much larger than the $\varepsilon$ above but still extremely small.

\section{Proof of the Main Lemma}

For simpler notation we translate the upper face of the considered cylinder to the plane $\pi(0)$. So here we assume that $Z=\operatorname{Cyl}(r, \gamma r, 0), \gamma=\beta-\alpha$.

We argue by contradiction; so we assume that $\mathcal{C}^{+}$is a packing of translates of $C$ with $T \cap \bigcup \mathcal{C}^{+}=\emptyset$ that misses less than an $\eta$ fraction (of the volume) of $Z$. We suppose that all cones in $\mathcal{C}^{+}$intersect $Z$.

We set $\rho=2 \sqrt{\eta} r$ and we let $V=\operatorname{Cyl}(\rho, \gamma r, \gamma r / 2)$ be a smaller cylinder in the lower half of $Z$.

Claim 4.1. There is a $C_{1} \in \mathcal{C}^{+}$intersecting $V$ such that $a\left(C_{1}\right)<\gamma r+2 \rho$.

Proof. By the choice of $\rho$, the cylinder $V$ has volume $2 \eta \operatorname{Vol} Z$, and so it is met by some element of $\mathcal{C}$, say by $C_{1}$. Since $C_{1}$ is disjoint from the axis $T$ of the cylinder $Z$, there exists a halfspace $H$ with $T$ on its boundary and disjoint from $C_{1}$. Since $\operatorname{Vol}(H \cap V) \geq \eta \operatorname{Vol} Z$, there exists another $C_{2} \in \mathcal{C}^{+}$intersecting $V$.

For contradiction let us suppose that both $a\left(C_{1}\right) \geq \gamma r+2 \rho$ and $a\left(C_{2}\right) \geq \gamma r+2 \rho$. Then both $C_{1}$ and $C_{2}$ intersect $\pi(\gamma r)$ and both are at a distance of at most $\rho$ from $T$, implying that

$$
\operatorname{dist}\left(\pi(\gamma r) \cap C_{1}, \pi(\gamma r) \cap C_{2}\right) \leq 2 \rho .
$$

However, by the avoidance lemma (Lemma 2.3)

$$
\operatorname{dist}\left(\pi(\gamma r) \cap C_{1}, \pi(\gamma r) \cap C_{2}\right)>2 \rho,
$$

a contradiction. Thus we have $a\left(C_{1}\right)<\gamma r+2 \rho$ or $a\left(C_{2}\right)<\gamma r+2 \rho$, and at least one of the cones $C_{1}$ and $C_{2}$ satisfies the requirements of the claim.

Now let $C_{1} \in \mathcal{C}^{+}$be as in the claim, and let us put

$$
a_{1}=\min \left(\gamma r, a\left(C_{1}\right)\right) .
$$

Since $C_{1}$ intersects $V$, we have $a_{1} \geq \gamma r / 2$. Let $C_{2}, \ldots, C_{m}$ be the cones in $\mathcal{C}^{+}$with $a_{i}=a\left(C_{i}\right) \leq a_{1}$, where the notation is chosen so that $a_{1} \geq a_{2} \geq \cdots \geq a_{m} \geq 0$. 
We denote by $\tilde{C}$ a general element of our packing $\mathcal{C}^{+}$. For every $\tilde{C} \in \mathcal{C}^{+}$different from $C_{1}, \ldots, C_{m}$ we have $a_{1}<a(\tilde{C})$. For $x \in[0, \alpha r)$ we define

$$
\tilde{C}(x)=\operatorname{Area}(\tilde{C} \cap \pi(x) \cap Z) .
$$

The function $C_{i}(\cdot):[0, \gamma r] \rightarrow \mathbf{R}$ is positive, continuous, and increasing on $\left[0, a_{i}\right]$, and is equal to zero on $\left(a_{i}, a_{1}\right)$ for $i=1, \ldots, m$. For $\tilde{C} \in \mathcal{C}^{+}$different from these $C_{i}$, the function $C(\cdot)$ is nonnegative, continuous, and nondecreasing on $\left[0, a_{1}\right]$.

We denote by $M$ the volume missed by $\mathcal{C}^{+}$from $Z$. Set $a_{m+1}=0$. Then

$$
\begin{aligned}
M & =\int_{0}^{\gamma r}\left(r^{2} \pi-\sum_{\tilde{C} \in \mathcal{C}^{+}} \tilde{C}(x)\right) d x \\
& \geq \int_{0}^{a_{1}}\left(r^{2} \pi-\sum_{\tilde{C} \in \mathcal{C}^{+}} \tilde{C}(x)\right) d x \\
& =\sum_{i=1}^{m} \int_{a_{i+1}}^{a_{i}}\left(r^{2} \pi-\sum_{\tilde{C} \in \mathcal{C}^{+}} \tilde{C}(x)\right) d x \\
& \geq \sum_{i=1}^{m} \int_{a_{i+1}}^{a_{i}}\left(\sum_{\tilde{C} \in \mathcal{C}^{+}} \tilde{C}\left(a_{i}\right)-\sum_{\tilde{C} \in \mathcal{C}^{+}} \tilde{C}(x)\right) d x \\
& \geq \sum_{i=1}^{m} \int_{a_{i+1}}^{a_{i}}\left(C_{1}\left(a_{i}\right)-C_{1}(x)\right) d x .
\end{aligned}
$$

Here the last inequality holds since $\tilde{C}\left(a_{i}\right) \geq \tilde{C}(x)$ for $x \in\left[a_{i}, a_{i+1}\right]$, and hence we can restrict the summation to the single cone $C_{1}$. The previous inequality follows from $\sum_{\tilde{C} \in \mathcal{C}^{+}} C(x) \leq r^{2} \pi$, which holds since $\mathcal{C}^{+}$is a packing. We need a simple claim, whose proof is postponed to the end of this section.

Claim 4.2. For $0 \leq x \leq y \leq a_{1}$ we have

$$
C_{1}(y)-C_{1}(x) \geq \frac{r}{4}(y-x) .
$$

We continue the last inequality for the missed volume $M$ :

$$
\begin{aligned}
M & \geq \sum_{i=1}^{m} \int_{a_{i+1}}^{a_{i}}\left(C_{1}\left(a_{i}\right)-C_{1}(x)\right) d x \\
& \geq \sum_{i=1}^{m} \int_{a_{i+1}}^{a_{i}} \frac{r}{4}\left(a_{i}-x\right) d x=\frac{r}{8} \sum_{i=1}^{m}\left(a_{i}-a_{i+1}\right)^{2} \\
& \geq \frac{r}{8} \cdot \frac{\left(\sum_{1}^{m}\left(a_{i}-a_{i+1}\right)\right)^{2}}{m}=\frac{r}{8} \cdot \frac{\left(a_{1}\right)^{2}}{m} \geq \frac{\gamma^{2} r^{3}}{32 m} .
\end{aligned}
$$


By now we are almost finished with the proof. First, all $C_{i}$ intersect the $\operatorname{disk} r D \subset \pi$, and, for each $i=1, \ldots, m$,

$$
a_{i} \leq a_{1}<\gamma r+2 \rho<\alpha r+2 \sqrt{\eta} r=3 \alpha r<2 r .
$$

Thus by the last part of Lemma 2.4 we have $m \leq 6$. Second, since Vol $Z=\gamma r^{3} \pi$, we have

$$
M \geq \frac{\gamma^{2} r^{3}}{32 m} \geq \frac{\gamma}{192 \pi} \operatorname{Vol} Z=\eta \operatorname{Vol} Z,
$$

contrary to our assumption that $M<\eta \mathrm{Vol} Z$.

Proof of Claim 4.2. We recall that $C_{1}$ is the cone in $\mathcal{C}^{+}$intersecting the smaller cylinder $V$, avoiding the axis $T$ (of $V$ and $Z$ ), and satisfying $a\left(C_{1}\right)<\gamma r+2 \rho$. We write $T_{1}$ for the axis of $C_{1}$. For $0 \leq x \leq a\left(C_{1}\right)$, we let $p_{0}(x)$ denote the point in the slice $C_{1} \cap \pi(x)$ nearest to $T$. Clearly, $p_{0}(x)$ is unique and lies on the boundary of $C_{1} \cap \pi(x)$. We denote by $T(x)$ the point $T \cap \pi(x)$, and $T_{1}(x)$ is the point $T_{1} \cap \pi(x)$. $\frac{1}{2}-\rho$.

It follows easily from $a\left(C_{1}\right)<\gamma r+2 \rho$ that $T_{1}$ is far from $T$ : their distance is at least

Since $C_{1}$ intersects $V$, we have $\left|T\left(a_{1}\right)-p_{0}\left(a_{1}\right)\right| \leq \rho$. The segment $\left[p_{0}\left(a_{1}\right), v_{1}\right]$, where $v_{1}$ is the tip of $C_{1}$, lies in $C_{1}$, and so, by Fact 2.1, the point $\left[p_{0}\left(a_{1}\right), v_{1}\right] \cap \pi(x) \in C_{1}$ is at distance at most $\rho+\left(a_{1}-x\right)$ from $T(x)$. This implies that, for all $x \in\left[0, a_{1}\right]$,

$$
\left|T(x)-p_{0}(x)\right| \leq \rho+\left(a_{1}-x\right) \leq 2 \sqrt{\eta} r+\gamma r<3 \alpha r .
$$

Further, for all $x \in\left[0, a_{1}\right], \pi(x) \cap \partial C_{1}$ is a closed convex curve in $\pi(x)$; see Fig. 4 . The part of this closed convex curve that lies in $Z$ consists of connected components; let $L(x)$ denote the component containing $p_{0}(x)$. Since $T_{1}$ is far from $T, C_{1} \cap \pi(x)$ cannot lie completely in $Z$. Thus $L(x)$ is a convex curve with two distinct endpoints. Consequently, the length $\ell(x)$ of $L(x)$ satisfies

$$
\ell(x) \geq 2(r-|T(x)-p(x)|) \geq 2(r-3 \alpha r) \geq \frac{3}{2} r .
$$

Let $p$ be an arbitrary point of the curve $L(x)$, and let $q$ be the intersection point of $\pi(y)$ and the line through $p$ and $v_{1}$. Further, let $C^{*}, L^{*}$, and $p^{*}$ denote the orthogonal projection of $C_{1} \cap \pi(x), L(x)$, and $p$, respectively, onto $\pi(y)$; see Fig. 5. We have

$$
C_{1}(y)-C_{1}(x)=\operatorname{Area}\left(\left(\left(C_{1} \cap \pi(y)\right) \backslash C^{*}\right) \cap Z\right) .
$$

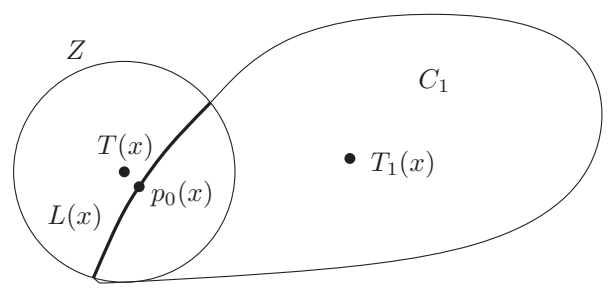

Fig. 4. Proof of Claim 4.2-the situation in the plane $\pi(x)$. 


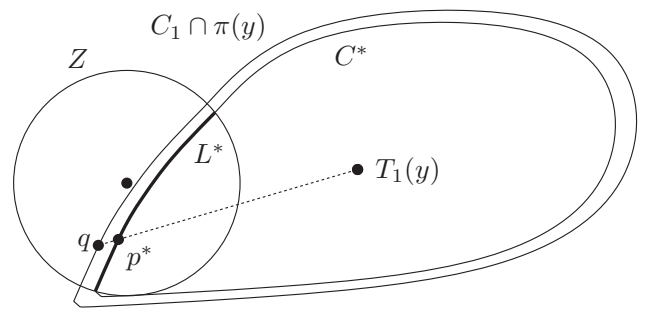

Fig. 5. Proof of Claim 4.2 continued.

Since $C^{*}$ is a homothetic copy of $C_{1} \cap \pi(y)$ with center of homothety $T_{1}(y)$, the points $q, p^{*}, T_{1}(y)$ are collinear. It follows from Fact 2.1 that $\left|q-p^{*}\right| \geq \frac{1}{2}(y-x)$.

Further, Fact 2.2 shows that the angle between the segment $\left[q, p^{*}\right]$ and the tangent line to $L^{*}$ at $p^{*}$ is at least $30^{\circ}$. Define

$$
F=\bigcup_{p \in L(x)}\left[q, p^{*}\right]
$$

It is now clear that

$$
\text { Area } F \geq \frac{1}{2}(y-x) \ell(x) \sin 30^{\circ}=\frac{1}{4}(y-x) \ell(x) .
$$

It is not hard to see that $F$ almost coincides with $\left(\left(C_{1} \cap \pi(x)\right) \backslash C^{*}\right) \cap Z$. More precisely, let $L^{\prime}$ be the set of those $p \in L(x)$ for which the segment $\left[q, p^{*}\right]$ is contained in $Z$. One can show readily that the length of $L^{\prime}$ is at least $\frac{2}{3} \ell(x)$; we omit the elementary details. Finally we have

$$
\begin{aligned}
C_{1}(y)-C_{1}(x) & =\operatorname{Area}\left(\left(\left(C_{1} \cap \pi(x)\right) \backslash C^{*}\right) \cap Z\right) \\
& \geq \frac{2}{3} \operatorname{Area} F \geq \frac{1}{6}(y-x) \ell(x) \\
& \geq \frac{1}{4}(y-x) r .
\end{aligned}
$$

\section{Proof of the Auxiliary Lemmas}

Proof of Lemma 2.3. The cone $C_{1}$ intersects the plane $\pi\left(a_{2}\right)$ and $C_{1} \cap \pi\left(a_{2}\right)$ is a homothetic copy of the base $B$. This homothetic copy and $B\left(C_{2}\right)$ are disjoint and so they can be separated in $\pi\left(a_{2}\right)$ by a line $\ell$. For $i=1,2$ let $\ell_{i}$ be the line that is the intersection of $\pi$ with the affine hull of $v\left(C_{i}\right)$ and $\ell$. The strip between $\ell_{1}$ and $\ell_{2}$ separates $\pi \cap C_{1}$ and $\pi \cap C_{2}$. Its width is at least $a_{2}$, as one can easily see using Fact 2.1.

Proof of Lemma 2.4. We show first that there is at most one cone $C^{*} \in \mathcal{C}^{+}$with $a\left(C^{*}\right)>2 r$. If there were two, $C_{1}$ and $C_{2}$, then

$$
\operatorname{dist}\left(C_{1} \cap \pi, C_{2} \cap \pi\right) \geq 2 r
$$

by the avoidance lemma (Lemma 2.3). However, since $r D$ lies in the $2 r$-neighborhood (in $\pi$ ) of $C_{1} \cap \pi, C_{2}$ cannot intersect $r D$, a contradiction. 
Next, let $C_{1}, \ldots, C_{m} \in \mathcal{C}^{+}$be the cones intersecting $r D$. We are done if $m \leq 1$. For $m \geq 2$ we may assume $a\left(C_{i}\right) \leq 2 r$ for all $i \geq 2$. For each $i=2, \ldots, m$ there is a point $p_{i} \in r D \cap \partial C_{i}$. Since for $i \geq 2, \pi \cap C_{i}$ is a copy of the base $B$ scaled by a factor between $1-2 r$ and 1, Fact 2.2 implies the existence of a planar wedge $K_{i} \subset \pi$, with apex at $p_{i}$ and angle $60^{\circ}$, such that $G_{i}=\left(p_{i}+\left(\frac{1}{2}-r\right) D\right) \cap K_{i}$ lies completely in $C_{i}$.

An elementary computation (using $r \leq \frac{1}{12}$ ) shows that $G_{i}$ intersects the boundary of the disk $\frac{1}{2} D$ in an arc longer than $0.15 \pi$. (We omit the details of this argument.) Since these arcs are disjoint, there are at most $\pi / 0.15 \pi=6.66 \ldots$ of them. Thus $m \leq 7$ follows.

Proof of Lemma 2.5. Let $C_{0}, C_{1}, \ldots, C_{m} \in \mathcal{C}$ be the cones intersecting the cylinder $\operatorname{Cyl}(R, \alpha R, 0)$ with $a_{1} \geq a_{2} \geq \cdots \geq a_{m}$.

We show first that $a_{i}<2 R$ for every $i$. This is satisfied if $a_{i} \leq \alpha R$, so suppose $a_{i}>\alpha R$. In this case $C_{i} \cap \pi(\alpha R)$ intersects the cylinder $\operatorname{Cyl}(R, \alpha R, 0)$ so the distance between $C_{i} \cap \pi(\alpha R)$ and $C_{0} \cap \pi(\alpha R)$ is at most $R-(\alpha / 2) R$. The avoidance lemma applied to $C_{i}$ and $C_{0}$ in the plane $\pi(\alpha R)$ shows that

$$
\operatorname{dist}\left(C_{i} \cap \pi(\alpha R), C_{0} \cap \pi(\alpha R)\right) \geq a_{i}-\alpha R .
$$

So we have $a_{i} \leq R+(\alpha / 2) R<2 R$.

With $a_{i}<2 R$ proved, Lemma 2.4 applies and shows that $m \leq 6$.

Next we want to define $r$ whose existence is stated in the lemma. If $a_{1} \leq \beta R$, then $r=R$ will clearly do. So we suppose $a_{1}>\beta R$.

We call an index $j \in\{1, \ldots, m-1\}$ a big drop if

$$
a_{j+1} \leq \frac{2 \beta}{2+\alpha} a_{j}
$$

First we assume that there is a big drop, and let $j$ be the first big drop (that is, no $i<j$ is a big drop). Then, for all $i<j$,

$$
a_{i+1}>\frac{2 \beta}{2+\alpha} a_{i}, \quad \text { implying } a_{j}>\left(\frac{2 \beta}{2+\alpha}\right)^{j-1} a_{1}>\left(\frac{2 \beta}{2+\alpha}\right)^{j-1} \beta R .
$$

In this case $r=2 a_{j} /(2+\alpha)$ will do. Indeed, for $i>j$ we have $a_{i} \leq a_{j+1} \leq \beta r$, and thus $C_{i}$ lies completely above the considered cylinder $\operatorname{Cyl}(r, \alpha r, \beta r)$. For $i \leq j$, the avoidance lemma (applied in $\pi(\alpha r)$ ) and Fact 2.1 show that $C_{i} \cap \pi(\alpha r)$ is at least at a distance of

$$
\left(a_{i}-\alpha r\right)+\frac{\alpha}{2} r \geq a_{j}-\frac{\alpha}{2} r=r
$$

from the axis of $C_{0}$. This implies that $C_{i}$ does not intersect the interior of $\operatorname{Cyl}(r, \alpha r, 0)$. Also,

$$
r>\left(\frac{2 \beta}{2+\alpha}\right)^{j} R \geq\left(\frac{2 \beta}{2+\alpha}\right)^{5} R
$$

since $j \leq m-1 \leq 5$. 
Next, we assume that there is no big drop. Then $r=2 a_{m} /(2+\alpha)$ will do. Indeed, in this case $C_{i}$ is disjoint from the interior of $\operatorname{Cyl}(r, \alpha r, 0)$ for each $i \geq 1$. This can be checked the same way as in the previous paragraph. Finally,

$$
r>\left(\frac{2 \beta}{2+\alpha}\right)^{m} R \geq\left(\frac{2 \beta}{2+\alpha}\right)^{6} R
$$

\section{Acknowledgments}

For their hospitality and support we thank CNRS, and the universities of Jussieu and Marne-la-Vallée where most of the research reported here took place. We are also grateful to W. Kuperberg for useful and inspiring comments and for careful reading of the manuscript.

\section{References}

1. U. Betke, M. Henk, Densest lattice packings of 3-polytopes, Comput. Geom. 16 (2000), 157-186.

2. A. Bezdek, On the density of packings of congruent bodies, in: F. Glatz ed., Lectures at the Hungarian Academy of Sciences, MTA Press, Budapest, 1998, pp. 117-126 (in Hungarian).

3. A. Bezdek, W. Kuperberg, Packing space with convex cones, Manuscript, 2006.

4. L. Danzer, B. Grunbaum, V. Klee, Helly's theorem and its relatives, in: V. Klee ed., Convexity, Proc. Symp. Pure Math., Vol. VII, AMS, Providence, RI, 1963, pp. 101-108.

5. W. Kuperberg, private communication (2001).

6. H. Minkowski, Dichteste gitterformige Lagerung kongruenter Körper, Nachr. K. Ges. Wiss. Göttingen, Math.-Phys. KL (1904), 311-355, also in: Gesammelte Abhandlungen, vol. II, pp. 3-42, Leipzig, 1911.

Received February 27, 2006, and in revised form May 6, 2006. Online publication July 13, 2007. 